\title{
A CENTRAL LIMIT THEOREM ON HEISENBERG TYPE GROUPS
}

\author{
PETER OHRING
}

(Communicated by Palle E. T. Jorgensen)

\begin{abstract}
Heisenberg type groups are a class of 2-step nilpotent Lie groups which have many similarities with Heisenberg groups. In this paper we consider a commutative Banach algebra of radial, bounded, Borel measures on such groups and prove a central limit theorem for measures in this algebra satisfying suitable growth conditions.
\end{abstract}

\section{INTRODUCTION}

One of the main themes in the area of nilpotent Lie group analysis in the past two decades has been the recognition of many parallels between analysis on Euclidean space related to the orthogonal group on the one hand, and analysis on the Heisenberg group and the unitary group on the other. $[\mathrm{FH}]$ is a very nice survey of these ideas.

Many of these parallels extend in one way or another to the class of Heisenberg type groups ( $H$-type groups). These groups were introduced by A. Kaplan in [Ka]. In fact, in this paper, Kaplan shows that the sublaplacian on these groups has a fundamental solution that is of the same form as the fundamental solution on the Heisenberg group $H_{n}$. (The result on $H_{n}$ is due to Folland [Fo], and possibly marks the beginning of the developments mentioned above.)

In this paper we introduce a commutative algebra of "radial", bounded, Borel measures on $H$-type groups. For probability measures in this algebra satisfying certain integrability conditions we prove a central limit theorem analogous to one of the Euclidean versions (Theorem 4.1).

This result and its proof are closely tied to the above-mentioned parallels. Our proof is based on the classical Fourier analysis proof and exploits the quite explicit formulas we have for the Gelfand transform of the above-mentioned algebra.

Central limit theorems on noncommutative Lie groups have appeared in the literature. Roynette [Ro] and Baldi, Bougerol, and Crepel [BBC] have proven

Received by the editors January 15, 1990.

1980 Mathematics Subject Classification (1985 Revision). Primary 22E27, 60B15; Secondary $43 \mathrm{~A} 80$. 
such theorems on motion groups. The latter, in fact, uses a harmonic analysis approach, not dissimilar from ours.

The structure of this paper is as follows. In $\S 1$ we recall the definition of a Heisenberg type group and some important aspects of harmonic analysis on these groups. $\S 2$ introduces the commutative algebra of "radial" measures and important facts related to the Gelfand theory of this algebra. In $\S 3$ we recall the properties of the heat semigroup and its image under the Gelfand transform. Finally $\S 4$ contains the central limit theorem and its proof.

\section{Preliminaries}

We begin this section by recalling the definition of a Heisenberg type group, following [ $\mathrm{Ka}, \mathrm{CH}]$.

Definition. A group of type $H$ is a connected, simply connected, real Lie Group whose Lie algebra is of type $H$. A Lie algebra, $\mathbf{n}$ is of type $H$ if $\mathbf{n}=\mathbf{v} \oplus \mathbf{z}$; $\mathbf{v}, \mathbf{z}$ real Euclidean spaces, with a Lie algebra structure such that $\mathbf{z}$ is the center of $\mathbf{n}$ for all $v \in \mathbf{v}$ of length one, $\operatorname{ad}_{v}$ is a surjective isometry of the orthogonal complement of ker $\operatorname{ad}_{v}$ in $\mathbf{v}$, onto $\mathbf{z}$.

Let $N$ be a type $H$ group and $\mathbf{n}$ its Lie algebra. We can define a mapping $j: \mathbf{z} \rightarrow \operatorname{End}(\mathbf{v})$ by

$$
\langle j(z) v, w\rangle=\langle z,[v, w]\rangle
$$

for $z \in \mathbf{z}, v, w \in \mathbf{v}$. It can be shown (cf. [K]) that

$$
|j(z) v|=|z||v| \text { and } j(z)^{2}=-|z|^{2} I .
$$

Thus if $\omega$ is a unit vector in $\mathbf{z}\left(\omega \in S_{\mathbf{z}}\right.$, ) then $j(\omega)$ defines a complex structure on $\mathbf{v}$. Let $\mathbf{v}_{\omega}$ be the space $\mathbf{v}$ equipped with the complex structure $j(\omega)$. Let $\{,\}_{\omega}$ denote the Hermitian form on $\mathbf{v}_{\omega}$ :

$$
\{v, w\}_{\omega}=\langle v, w\rangle+i\langle j(\omega) v, w\rangle=\langle v, w\rangle+i\langle\omega,[v, w]\rangle .
$$

$j(\omega)$ nondegenerate implies that $\mathbf{v}$ is even dimensional, say $\operatorname{dim} \mathbf{v}=2 n$.

The representation theory of type $H$ groups is well known, (see [KR, $\mathrm{CH}]$ ), and is very similar to the Heisenberg group: There are basically two types of irreducible unitary representations, one-dimensional characters, and infinitedimensional representations. The characters correspond to the representations which are trivial on the center,

$$
\chi_{w}(v, z)=e^{i \operatorname{Re}\{v, w\}}
$$

where $z \in \mathbf{z}, v, w \in \mathbf{v}$. The infinite-dimensional representations can be parametrized by $\mathbf{z}-\{0\}$ which we identify with $\mathbf{R}^{+} \times S_{\mathbf{z}}$. Let $\sigma_{\nu, \omega}$ denote the irreducible representation of $\mathbf{n}$ corresponding to $(\nu, \omega) \in \mathbf{R}^{+} \times S_{\mathbf{z}} \cdot \sigma_{\nu, \omega}$ acts on $H_{\nu, \omega}$, the space of holomorphic functions on $\mathbf{v}_{\omega}$ such that

$$
\|\xi\|_{\nu}^{2}=\int_{\mathbf{v}}|\xi(v)|^{2} e^{-2 \nu|v|^{2}} d v<\infty
$$


and $\|\xi\|_{v}$ is the norm of $\xi$ in $H_{\nu, \omega}$. We have

$$
\left[\sigma_{\nu, \omega}(v, z) \xi\right](w)=e^{-\nu\left(|v|^{2}+2\{w, v\}_{\omega}+i\langle z, \omega\rangle\right)} \xi(\nu+\omega) .
$$

The Plancherel measure on $\mathbf{R}^{+} \times S_{\mathbf{z}}$ is $c \nu^{n+q-1} d \nu d \omega$ where $c$ is a constant related to the surface measure of $S_{\mathbf{z}}, q$ is the dimension of $\mathbf{z}$, and $d \omega$ is the normalized surface measure on $S_{\mathbf{z}}$. (For a discussion of the Plancherel formula see $[\mathrm{CH}]$.)

There is a natural dilation structure on $N$. For $s>0$ define $\delta_{s}(v, z)=$ $\left(s v, s^{2} z\right) . \delta_{s}$ is an automorphism of $N$ and so

$$
\left(\delta_{s} f\right)(v, z)=s^{2(n+q)} f\left(s v, s^{2} z\right)
$$

defines an automorphism of $L^{1}(N)$. This automorphism extends to $M_{b}(N)$, the bounded Borel measures on $N$, by duality.

\section{V-RADIAL MEASURES ON $H$-TYPE GROUPS}

Let $U_{\omega}$ denote the unitary group on $\mathbf{v}_{\omega} \cdot U_{\omega}$ acts naturally on $H_{\nu, \omega}$. For $\xi \in H_{\nu, \omega}, v \in \mathbf{v}, A \in U_{\omega}$ we have

$$
A \xi(v)=\xi(A v) .
$$

It is a direct consequence of (1.2) and (2.1) that

$$
A \sigma_{\nu, \omega}(A v, z)=\sigma_{\nu \omega}(v, z) A .
$$

$\sigma_{\nu, \omega}$ lifts to a representation of $M_{b}(N)$. For $\mu \in M_{b}(N)$ we have

$$
\sigma_{\nu, \omega}(\mu)=\int_{\mathbf{v}} \int_{\mathbf{z}} \sigma_{\nu, \omega}(v, z) d \mu(v, z) .
$$

$\mu \in M_{b}(N)$ is said to be $\mathbf{v}$ radial if $d \mu(A v, z)=d \mu(v, z)$ for all $A \in O(\mathbf{v})$. Let $M_{b}(N)^{\natural}$ (respectively $L^{1}(N)^{\natural}$ ) denote the closed Banach algebra generated by the v-radial measures, (respectively v-radial $L^{1}$ functions).

$M_{b}(N)^{\natural}$ is an example of a Gelfand-Levitan space (cf. [Ge]). Such spaces are generalizations of Gelfand pairs. $L^{1}(N)^{\natural}$ does not come from a Gelfand pair unless $N$ is the Heisenberg group, for in that case the v-radial $L^{1}$ functions form a subalgebra of $L^{1}$.

Lemma 2.1. If $\mu \in M_{b}(N)^{\natural}$ and $A \in U_{\omega}$ then

$$
A \sigma_{\nu, \omega}(\mu) \xi(w)=\sigma_{\nu, \omega} A \xi(w)
$$

where $\xi \in H_{\nu, \omega}$.

Proof. It suffices to prove the lemma for $\mu$ v-radial as the v-radial measures generate $M_{b}(N)^{\natural} . A \in U_{\omega}$ implies that $A \in O(v)$. Since $\mu$ is $v$-radial (2.2) 
implies that

$$
\begin{aligned}
\sigma_{\nu, \omega}(\mu) \xi(A w) & =\iint\left[\sigma_{\nu, \omega}(v, z) \xi\right](A w) d \mu(v, z) \\
& =\iint A\left[\sigma_{\nu, \omega}(A v, z) \xi\right](w) d \mu(A v, z) \\
& =\iint\left[\sigma_{\nu, \omega}(v, z) A \xi\right](w) d \mu(v, z) \\
& =\left[\sigma_{\nu, \omega}(\mu) A \xi\right](w) .
\end{aligned}
$$

For $\omega \in S_{\mathbf{z}}$ we can identify $\mathbf{v}_{\omega}$ with $\mathbf{C}^{n}$ in such a way that $\{v, w\}_{\omega}$ becomes the usual Hermitian form on $\mathbf{C}^{n}$. We introduce the usual orthonormal basis of monomials on $H_{\nu, \omega}$ : For $\mathbf{m} \in \mathbf{N}_{0}^{n}, \mathbf{N}_{0}=(0,1,2, \ldots)$ let $e_{\mathbf{m}}=e_{\mathbf{m}, \nu, \omega}$ in $H_{\nu, \omega}$ be

$$
e_{\mathbf{m}}(w)=(2 \nu / \pi)^{n / 2}(2 \nu)^{|\mathbf{m}| / 2}(\mathbf{m} !)^{-1 / 2} w^{\mathbf{m}},
$$

where $|\mathbf{m}|=m_{1}+m_{2}+\cdots+m_{n}, \mathbf{m} !=m_{1} ! m_{2} ! \cdots m_{n} !$ and $w^{\mathbf{m}}=w_{1}^{m_{1}} w_{2}^{m_{2}} \cdots w_{n}^{m_{n}}$. Let $\mathscr{P}_{m}$ denote the subspace of homogeneous polynomials of degree $m$. $U_{\omega}$ acts irreducibly on $\mathscr{P}_{m}$ and it is well known that the action of $U_{\omega}$ on $H_{\nu, \omega}$ is multiplicity free. Thus if $\mu \in M_{b}(N)^{\natural}$, Lemma 2.1 implies that $\sigma_{\nu, \omega}(\mu)$ preserves each $\mathscr{P}_{m}$. By Schur's lemma $\sigma_{\nu, \omega}$ acts on $\mathscr{P}_{m}$ by a scalar. (This scalar is just $\left\langle\sigma_{\nu, \omega}(\mu) e_{\mathrm{m}}, e_{\mathbf{m}}\right\rangle$ for any $\mathbf{m}$ with $|\mathbf{m}|=m$.) It follows that $M_{b}(N)^{\natural}$ is a commutative Banach algebra.

We will denote by $\widehat{\mu}(\nu, \omega, \mathbf{m})$ the matrix coefficient $\left\langle\sigma_{\nu, \omega}(\mu) e_{\mathrm{m}}, e_{\mathrm{m}}\right\rangle$ and write

$$
\chi_{\nu, \omega, m}(\mu)=\widehat{\mu}(\nu, \omega, \mathbf{m}) \quad \text { where }|\mathbf{m}|=m
$$

We also write

$$
\chi_{\rho}(\mu)=\widehat{\mu}(\rho)=\iint \chi_{\omega}(v, z) d \mu(v, z),
$$

where $\rho=|w|$ and $\chi_{w}$ is as in (1.1).

We now present three propositions which sum up some of the most important facts related to the commutative Banach algebras, $M_{b}(N)^{\natural}$ and $L^{1}(N)^{\natural}$. We omit the proofs as they are completely analogous to the proofs on the $2 n+1$ dimensional Heisenberg group. In this setting these results are well known and can be found in $[\mathrm{FH}]$. (See also [HR] and [BJR].)

Proposition 2.2. The spectrum of $L^{1}(N)^{\natural}$, i.e. the set of nonzero multiplicative linear functionals on $L^{1}(N)^{\natural}$, is equal to

$$
\left\{\chi_{\nu, \omega, m}: \nu \in \mathbf{R}^{+}, \omega \in S_{\mathbf{z}}, m \in \mathbf{N}_{0}\right\} \cup\left\{\chi_{\rho}: \rho \geq 0\right\} \text {. }
$$

Proposition 2.3. If $\mu \in M_{b}(N)^{\natural}$ then

$$
\widehat{\mu}(\nu, \omega, \mathbf{m})=\iint e^{-i \nu\langle z, \omega\rangle-\nu|v|^{2}} \prod_{j=1}^{n} L_{m_{j}}\left(2 \nu\left|v_{j}\right|^{2}\right) d \mu(v, z)
$$


where $|\mathbf{m}|=m$ and $L_{k}$ is the Laguerre polynomial of degree $k$; that is,

$$
L_{k}(x)=\sum_{j=0}^{k}\left(\begin{array}{l}
k \\
j
\end{array}\right) \frac{(-x)^{j}}{j !} .
$$

Proposition 2.4. The set of functions in $L^{1}(N)^{\natural}$ whose Gelfand transform $\hat{f}$ has compact support in $\mathscr{M}$ is dense in $L^{1}(N)^{\natural}$.

The rest of this section is devoted to some rather standard facts related to the Gelfand theory of $L^{1}(N)^{\natural}$ that will be needed in $\S 4$. Let $\mathscr{M}$ denote the Gelfand space of $L^{1}(N)^{\natural}$. For $f \in \mathscr{S}(N)$ the inversion formula on $N$ takes the form

$$
f(v, z)=c \int_{S_{\mathbf{z}}} \int_{R^{+}} T_{r}\left(\sigma_{\nu, \omega}(f) \sigma_{\nu, \omega}(v, z)^{*}\right) \nu^{n+q-1} d \nu d \omega .
$$

If, furthermore, $f \in L^{1}(N)^{\natural}$ then

$$
f(v, z)=c \int_{S_{\mathbf{z}}} \int_{R^{+}} \sum_{\mathbf{m}} \widehat{f}(\nu, \omega, m)\left\langle\sigma_{\nu, \omega}(v, z)^{*} e_{\mathbf{m}}, e_{\mathbf{m}}\right\rangle \nu^{n+q-1} d \nu d \omega .
$$

We will denote by $I^{k}(N)^{\natural}$ the ideal in $L^{1}(N)^{\natural}$ of functions whose Gelfand transform has compact support. By Proposition $2.4 I^{k}(N)^{\natural}$ is dense in $L^{1}(N)^{\natural}$.

Lemma 2.5. $I^{k}(N)^{\natural} \subset C_{0}(N)$ where $C_{0}(N)$ denotes continuous functions on $N$ that vanish at infinity.

Proof. For $\psi \in L^{1}(\mathscr{M})$ (all $L^{p}(\mathscr{M})$ spaces with respect to the Plancherel measure), set

$$
\left(\mathscr{G}^{-1} \psi\right)(v, z)=c \int_{S_{z}} \int_{R_{+}} \sum \psi(\nu, \omega, m)\left\langle\sigma_{\nu, \omega}(v, z)^{*} e_{\mathrm{m}}, e_{\mathrm{m}}\right\rangle \nu^{n+q-1} d \nu d \omega .
$$

Let $\varphi \in I^{k}(N)^{\natural} . \mathscr{G}^{-1} \hat{\varphi}$ is continuous on $N$ since $\hat{\varphi}$ has compact support. Let $\left\{\varphi_{n}\right\}$ be a sequence of Schwartz functions in $L^{1}(N)^{\natural} \quad\left(\varphi_{n} \in \mathscr{S}(N)^{\natural}\right)$ converging to $\varphi$ in $L^{1}(N)^{\natural}$. Thus $\widehat{\varphi}_{n}$ converges uniformly to $\widehat{\varphi}$. Furthermore the inversion formula hold for $\varphi_{n}$ :

$$
\varphi_{n}(v, z)=\left(\mathscr{G}^{-1} \widehat{\varphi}_{n}\right)(v, z)
$$

It easily follows that $\varphi=\mathscr{G}^{-1} \hat{\varphi}$; hence $\varphi$ is continuous. The decay in the $v$ coordinate of $\varphi$ at infinity follows from the rapid decay at infinity of

$$
e^{-\nu|v|^{2}} \prod_{j=1}^{m} L_{m_{j}}\left(2 \nu\left|v_{j}\right|^{2}\right)
$$

and the dominated convergence theorem. The decay in the $z$ coordinate follows from the Riemann-Lebesgue lemma. 
The Plancherel theorem in this setting has it that for $f, g \in \mathscr{S}(N)^{\natural}$,

$$
\begin{aligned}
\langle f, g\rangle & =\int_{\mathscr{S}_{\mathbf{z}}} \int_{\mathbf{R}^{+}} \sum \widehat{f}(\nu, \omega, m) \overline{\widehat{g}(\nu, \omega, m)} \nu^{n+q-1} d \nu d \omega \\
& \equiv\langle\widehat{f}, \widehat{g}\rangle .
\end{aligned}
$$

The image of $\mathscr{S}(N)^{\natural}$ under the Gelfand transform is dense in $C_{0}(\mathscr{M})$ by the Stone-Weierstrass theorem. Hence this image is dense in $L^{2}(\mathscr{M})$. Together with (2.5) this implies that $f \rightarrow \widehat{f}$ extends to an isometry of $L^{2}(N)^{\natural}$ onto $L^{2}(\mathscr{M})$. (Here, $L^{2}(N)^{\natural}$ denotes the $L^{2}$ closure of $S(N)^{\natural}$.)

Lemma 2.6. Let $\mu \in M_{b}(N)^{\natural}$ and $\varphi \in I^{k}(N)^{\natural}$. Then

$$
\langle\mu, \varphi\rangle=\langle\widehat{\mu}, \widehat{\varphi}\rangle \text {. }
$$

Proof. Let $\left\{g_{n}\right\}$ be an approximate identity in $L^{1}(N)^{\natural}$ such that $g_{n}$ is also in $L^{2}(N)$ for $n=0,1,2, \ldots$ We have $\mu * g_{n} \in L^{2}(N)^{\natural}$ and $\left(\mu * g_{n}\right)^{\wedge}$ converges uniformly to $\widehat{\mu}$. Hence it follows from the dominated convergence theorem and the Plancherel formula (2.5) that

$$
\langle\mu, \varphi\rangle=\lim _{n \rightarrow \infty}\left\langle\mu * g_{n}, \varphi\right\rangle=\lim _{n \rightarrow \infty}\left\langle\widehat{\mu} \cdot \widehat{g}_{n}, \widehat{\varphi}\right\rangle=\langle\widehat{\mu}, \widehat{\varphi}\rangle .
$$

\section{THE HEAT SEMIGROUP}

Let $\Delta$ be the usual Laplacian on $\mathbf{v}$. We will denote by $\left\{p_{t}\right\}_{t>0}$ the semigroup of solutions of the heat equation corresponding to $\Delta$ on the group $N$. The following properties of $p_{t}$ are well known (cf. [Hu]):

(i) $p_{t}>0$;

(ii) $p_{t}$ is v-radial and in $C^{\infty}(N)$;

(iii) $\iint p_{t}(v, z) d v d z=1$.

We would like to compute $\widehat{p}_{t}(\nu, \omega, m)$ explicitly. This can be done easily upon noting that $\sigma_{\nu, \omega}$ is essentially the representation of a $2 n+1$ dimensional subgroup of $N$ that is isomorphic to $H_{n}$. To see this note that the orthogonal complement $\omega^{\perp}$, of $\omega$ in $\mathbf{z}$, is contained in $\operatorname{ker}\left(\sigma_{\nu, \omega}\right)$. Hence $\sigma_{\nu, \omega}$ factors through to a representation of $N / \exp \left(\omega^{+}\right)$. Since $j(\omega)$ is nondegenerate this quotient group is isomorphic to $H_{n}$.

Viewed in this way we can use the well-known fact (cf. [Ta]) that $\sigma_{\nu, \omega}(-\Delta)$ is diagonalized by $\left\{e_{m}\right\}$ and

$$
\sigma_{\nu, \omega}(-\Delta) e_{\mathrm{m}}=(2|\mathrm{~m}|+n) e_{\mathrm{m}} .
$$

It is another well-known result (cf. [Ta]) that

$$
\sigma_{\nu, \omega}\left(p_{t}\right)=e^{-t \sigma_{\nu, \omega}(-\Delta)}=e^{t \sigma_{\nu, \omega}(\Delta)} .
$$

It follows from (3.1) and (3.2) that

$$
\widehat{p}_{t}(\nu, \omega, \mathbf{m})=e^{-t \nu(2|\mathbf{m}|+n)} .
$$




\section{MAIN THEOREM}

Let $\operatorname{Co}(N)^{\natural}$ denote the uniform closure of $I^{k}(N)^{\natural}$. The main result of this paper is the following central limit theorem.

Theorem 4.1. Let $\mu \in M^{1}(N)^{\natural}$ be a probability measure such that

(i) $\iint\left\langle z, z^{\prime}\right\rangle d \mu(v, z)=0$ for all $z^{\prime} \in \mathbf{z}$,

(ii) $\iint e^{a|v|^{2}} d \mu(v, z)<\infty$ for some $a>0$,

(iii) $\iint|v|^{2} d \mu(v, z)=n$.

Then $\left(\delta_{\sqrt{k}}(\mu)\right)^{* k}$ converges weakly to $p_{1}$, i.e.

$$
\left\langle\left(\delta_{\sqrt{k}}(\mu)\right)^{* k}, \varphi\right\rangle \rightarrow\left\langle p_{1}, \varphi\right\rangle \text { for all } \varphi \in C_{0}(N)^{\natural},
$$

where $p_{1}$ denotes the element of the semigroup $\left\{p_{t}\right\}$ corresponding to $t=1$. (See § 3.)

Proof. The integrability hypothesis (ii) above insures that (2.3) makes sense not only for $\nu>0$, but on a neighborhood of $\nu=0$. Using (2.3) it is easily computed that $\widehat{\mu}(0, \omega, \mathbf{m})=1$ and that

$$
\begin{aligned}
\frac{d}{d \nu} \widehat{\mu}(0, \omega, \mathbf{m}) & =\iint\left[-i\langle z, \omega\rangle-|v|^{2}-\sum_{j=1}^{n} 2 m_{j}\left|v_{j}\right|^{2}\right] d \mu(v, z) \\
& =-\iint \sum_{j=1}^{n}\left(2 m_{j}+1\right)\left|v_{j}\right|^{2} d \mu(v, z) \\
& =-\sum_{j=1}^{n}\left(2 m_{j}+n\right) \iint\left|v_{j}\right|^{2} d \mu(v, z) \\
& =-(2|m|+n) .
\end{aligned}
$$

The Taylor-Maclaurin expansion of $\widehat{\mu}(\nu, \omega, m)$ in $\nu$ is

$$
\widehat{\mu}(\nu, \omega, \mathbf{m})=1-(2|\mathbf{m}|+n) \nu+o(\nu) .
$$

Now from (1.3) and (2.3) it follows that

$$
\left(\delta_{\sqrt{k}}(\mu)\right)^{\curlywedge}(\nu, \omega, \mathbf{m})=\widehat{\mu}(\nu / k, \omega, \mathbf{m}) .
$$

If we set $\mu_{k}=\left(\delta_{\sqrt{k}}(\mu)\right)^{* k}$ then

$$
\mu_{k}(\nu, \omega, \mathbf{m})=(1-(2|\mathbf{m}|+n) \nu / k+o(\nu / k))^{k} .
$$

This, together with (3.3), immediately yields

$$
\lim _{k \rightarrow \infty} \widehat{\mu}_{k}(\nu, \omega, \mathbf{m})=\widehat{p}_{1}(\nu, \omega, \mathbf{m}) .
$$

We now need to go from pointwise convergence of the transforms to weak convergence on the group. First we will assume that $\varphi \in I^{k}(N)^{\natural}$. Lemma (2.6) implies that

$$
\lim _{k \rightarrow \infty}\left\langle\mu_{k}, \varphi\right\rangle=\lim _{k \rightarrow \infty}\left\langle\widehat{\mu}_{k}, \widehat{\varphi}\right\rangle=\left\langle p_{1}, \varphi\right\rangle,
$$


provided we can justify the second equality above. This follows from the dominated convergence theorem as $\widehat{\mu}_{k}$ is dominated by 1 and $\widehat{\varphi} \in C_{0}(\mathscr{M})$. The result extends to all of $C_{0}(N)^{\natural}$ by a $3 \varepsilon$ argument using the uniform boundedness of $\left\{\mu_{k}\right\}$.

Remark. It would nice to do away with hypothesis (ii) in the statement of the theorem. Such an integrability condition does not arise in either the classical case or on the motion group (cf. [Ro, BBC]). At the moment the author does not see a way to avoid it.

\section{ACKNOWLEDGMENT}

We would like to thank Olivier Gebuhrer and Joe Jenkins for many helpful discussions during the preparation of this paper.

\section{REFERENCES}

[BBC] P. Baldi, P. Bougerol, and P. Crepel, Théorème central limite local sur les déplacements de $\mathbf{R}^{d}$, C. R. Acad. Sci. Paris Sér. A 283 (1976), 53-55.

[BJR] C. Benson, J. Jenkins, and G. Ratcliff, On Gelfand pairs associated with solvable Lie groups, Trans. Amer. Math. Soc. 321 (1990), 85-116.

[CH] M. Cowling and U. Haagerup, Completely bounded multipliers of the Fourier algebra of a simple Lie group of real rank one, Invent. Math. 96 (1989), 507-549.

[FH] J. Faraut and Khélifa Harzalleh, Deux cours d'analyse harmonique, Birkhäuser, Boston, 1987.

[Fo] G. Folland, A fundamental solution for a subelliptic operator, Bull. Amer. Math. Soc. 79 (1973).

[Ge] O. Gebuhrer, Analyse harmonique sur les espaces de Gelfand Levitan et applications à la théorie des semi-groupes de convolution, Thèse d'etat, Universite Louis Pasteur, Strasbourg, 1989.

[HR] H. Hulanicki and F. Ricci, A tauberian theorem and tangential convergence of bounded harmonic functions on balls in $\mathbf{C}^{2}$, Invent. Math. 62 (1980), 325-331.

[Hu] A. Hulanicki, $A$ class of convolution semi-groups of measures on a Lie group, Lecture Notes in Math., vol. 828, Springer-Verlag, 1980, pp. 82-101.

[Ka] A. Kaplan, Fundamental solutions for a class of hypoelliptic PDE generated by composition of quadratic forms, Trans. Amer. Math. Soc. 258 (1980), 147-153.

[KR] A. Kaplan and F. Ricci, Harmonic analysis on groups of Heisenberg type, Harmonic analysis, Lecture Notes in Math., vol. 992, Springer-Verlag, Berlin, 1983, pp. 416-435.

[Ro] B. Roynette, Théorème central limite pour le group des déplacements de $\mathbf{R}^{d}$, Ann. Inst. Henri Poincaré, X (1974), 391-398.

[Ta] M. Taylor, Noncommutative harmonic analysis, Amer. Math. Soc., Providence, RI, 1986.

Department of Mathematics and Statistics, State University of New York, Albany, NEW YORK 12222

Current address: Department of Mathematics, Bowdoin College, Brunswick, Maine 04011 